\title{
Study the Characteristic of the Coupling Parameter $(\Gamma)$ in Dusty Plasma by Computer modeling
}

\author{
Hamid H. Murbat* Bayan G. Ali*
}

Date of acceptance $28 / 2 / 2010$

\begin{abstract}
Computer modeling has been used to investing the Coulomb coupling parameter $\Gamma$. The effects of the structure parameter $K$, grain charge $Z$, plasma density $N$, temperature dust grain $T_{d}$, on the Coulomb coupling parameter had been studied. It was seen that the $\Gamma$ was increasing with increasing $Z$ and $N$, and decrease with increasing $K$ and $T$. Also the critical value of $\Gamma$ that the phase transfer of the plasma state from liquid to solid was studied.
\end{abstract}

Key words: Coulomb coupling parameter, dust grain, dust charge, structure parameter, phase transition, screening length, radius grain, potential energy .

\section{Introduction}

Dusty plasmas (also known as complex plasmas) are ordinary plasmas with embedded solid particles. The particles can be made of either dielectric or conducting materials, and can have any shape. The typical size range is anywhere from $100 \mathrm{~nm}$ up to say $100 \mu \mathrm{m}$. Dust particles that are added to plasmas for experimental studies, tend to be glass or plastic particles(melamine formaldehyde is a commonly used particle) and are spherical with a very narrow distribution of diameters. Dusty plasmas are ubiquitous in the universe; examples are protoplanetary and solar nebulae, molecular clouds, supernova explosions, interplanetary medium, circumsolar rings, and asteroids. Dust is important in plasmas because it becomes charged. It can be charged by collecting electrons and ions from the plasma, or, in the presence of UV radiation, it can be charged by photoelectric emission. The dust in typical laboratory dusty plasmas is negatively charged, due to the fact that the electrons move about more swiftly than the ions. A 1 micron radius dust particle in a plasma with an electron temperature of a few $\mathrm{eV}$, will have a charge corresponding to a few thousand electron charge, with a resulting charge to mass ratio, $\mathrm{Q} / \mathrm{m}<1$.

One of the most interesting properties of a dusty plasma is related to their relatively large value of the charge on the particles. Since Q/e can be $\sim$, the interaction between dust grains can be very strong. As a result, dusty plasmas are often in the strongly coupled state, i.e., the ratio of. Coulomb potential energy to thermal kinetic energy can be much greater than one. In this state, the dust particles form ordered solid-like structures, called Coulomb solids or crystals. [1-4]

\section{Coupling Parameter}

The criteria of complex plasma condensation should depend strongly on the effect of screening. We demonstrate here that large charges (of the values of interest for the plasma condensation ) are screened differently than small charges (of the order of a single electron charge) in ordinary

\footnotetext{
* Physics Department, College of Science for Women, University of Baghdad.
} 
plasma. For small charges the Boltzman density distribution of the plasma polarization charges around then is approximated by the first terms of expansion of the potential $\mathrm{n}_{\mathrm{o}} \exp (-$ $\left.\mathrm{e} \varphi / \mathrm{T}_{\mathrm{e}}\right) \approx \mathrm{n}_{\mathrm{o}}\left(1-\mathrm{e} \varphi / \mathrm{T}_{\mathrm{e}}\right)$ and $\mathrm{n}_{\mathrm{o}} \exp (\mathrm{e} \varphi /$ $\left.\mathrm{T}_{\mathrm{i}}\right) \approx \mathrm{n}_{\mathrm{o}}\left(1+\mathrm{e} \varphi / \mathrm{T}_{\mathrm{i}}\right)$ for electrons and ions, respectively. The Poisson's equation for the dust charge screening is then reduced to a linear equation for the screening factor:

$$
\begin{aligned}
& \phi=\frac{-Z_{d} e}{r} \psi(r) \\
& \frac{d^{2} \psi}{d r^{2}}=\frac{\psi}{\lambda_{D}^{2}}
\end{aligned}
$$

$\frac{1}{\lambda_{D}^{2}}=4 \pi n_{o} e^{2}\left(\frac{1}{T_{i}}+\frac{1}{T_{e}}\right)$

where $\lambda_{\mathrm{D}}$ is the Debye screening length. By assuming that both the distance from the grain $r$ and the grain size are given in units of Debye screening length ((thus $r$ corresponds to $r / \lambda_{\mathrm{D}}$ and corresponds to $\left.a / \lambda_{\mathrm{D}}\right)$, yielding the exponential screening factor (sometimes called "Yukawa screening )

$$
\begin{gathered}
\psi=\exp (-(r-a)) \\
a<<1 .
\end{gathered}
$$

It is not correct to consider the exponential decrease of the potential as fast if we are interested in distances of the order of the screening distance. For example, at 4.6 screening distance the interaction decreases only by a factor of 100 . Since the Coulomb interaction is huge and $\mathrm{T}$ can be of the order of $10^{4}$, a decrease in the interaction by a factor of 100 still corresponds to strong coupling $\Gamma \gg>1$. Defining for the screened Coulomb interaction the dimensionless parameter

$$
K \equiv \frac{1}{N_{d}^{1 / 3} \lambda_{D}},
$$

we find

$\Gamma=\frac{Q^{2}\left(4 \pi N_{d} / 3\right)^{1 / 3}}{4 \pi \varepsilon_{o} T_{d}} \exp (-K)$

This exponential factor shows the importance of screening for strong coupling and plasma condensation.[5]

2. The state of the problem

Such a system can be realized, for example, within a highly evolved star, where the strongly degenerate electron gas creates a neutralizing background to the Coulomb interaction of ions. Then strength of the ionic interaction can be characterized by parameter equation (6). It is generally accepted to call the system nonideal, if $\Gamma \approx 1$ and strongly nonideal or strongly coupled at $\Gamma>1$

The dusty plasma contains electrons, ions and the charged dust grains. In the plasma any charge is screened by free charges of other sign. Therefore, one-component approach is incorrect. Ikezi [6] suggested considering the screening of charges in the equation for coupling parameter, using the Debye-Hückel or Yukawa potential for calculation of the potential energy of grains interaction. Then the coupling parameter for the dust grains subsystem can be presented as follows:

$\Gamma=\frac{\left(e Z_{d}\right)^{2}}{4 \pi \varepsilon_{o} R_{W} T_{d}} \exp (-K)$.

where $K=R_{W} / \lambda_{D}$ is the structural parameter, $r_{D}$ is the screening length, $T_{d}$ is the temperature of dust grains. Plasma crystals are formed at value $\Gamma>$ $\Gamma_{\mathrm{c}}$ where $\Gamma_{\mathrm{c}}$ is some critical value of the coupling parameter. Numerical calculation for one-component plasma give the value $\Gamma_{\mathrm{c}} \sim 170$.

The phase diagram of crystallization in coordinates $\Gamma-K$, was gained in the papers [8-11] by means of numerical modeling, but it (fra)s a 
different value if plasma shielding effects are considered. Under normal circumstances for ordinary ion/electron plasma. $\Gamma$ is much smaller than $\Gamma_{\mathrm{c}}$.Hence, the gaseous state normally prevails and we are most familiar with this plasma regime. The $\Gamma<<\Gamma_{\mathrm{c}}$ condition defines the weak coupling regime. However, in a dusty plasma, a dust grain can acquire a large amount of charge, i.e., $Q=e Z$ where $Z$ can be very large $\left(\sim 10^{3}-10^{5}\right)$. Under such circumstances it is possible for $\Gamma$ to exceed $\Gamma_{\mathrm{c}}$ for ordinary dust density and temperature values. This $\left(\Gamma>\Gamma_{\mathrm{c}}\right)$ condition defines the strong coupling regime where a solid- like behavior, such as Coulomb crystals, is manifested. For intermediate values of $\Gamma$ a liquid -like state exists.

Thus it is noted that the coupling parameter in the form of equation (7) provides a better description of a phase transition

\section{Results and Discussion}

Computer modeling has been used to investigate. Figures (1-3) shows the Coulomb coupling parameter $(\Gamma)$ as a function of the $(\Gamma)$ was decreased structural parameter $(K)$ for three different value of grain charge, it can be shown that with increasing $(K)$. This is expected because the increasing of $(K)$ mean increasing interparticle distance and decreasing of screening length $\left(r_{D}\right)\left(K=a / \mathrm{r}_{\mathrm{D}}\right)$. Hence the particles would gain more mobility to move. It can be shown that $(\Gamma)$ approach to zero at large values of $(K)$. Fig.(1) shows the Coulomb coupling parameter $(\Gamma)$ as a function of structure parameter $(K)$ for three different values of the charge of the grain $(Z)$, and constant $\left(T_{d}=0.025 \mathrm{eV}\right)$ and plasma density $\left(N=10^{10} \mathrm{~m}^{-3}\right)$. It can be shown that the coulomb coupling parameter was increased with increasing the charge grain because of increasing of $(Z)$ means that increasing the potential energy of the grain causing of increasing $(\Gamma)$. Same reason can be said about figures $(8,11)$. Also it can be seen that the critical value of $(\Gamma)=170$ occurs at $(Z=1000), K \approx 0.2)$ and $(Z)=$ $1500,(K \approx 0.9)$.

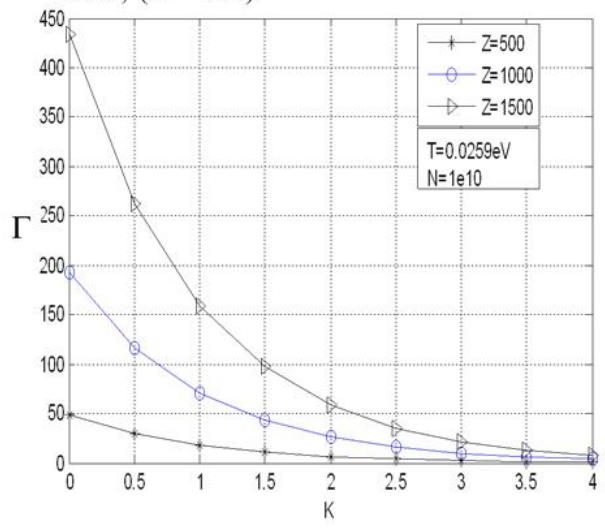

Fig.1 ( $\Gamma)$ as a function of $(K)$ for different value of $(Z)$.

Fig.(2) shows $(\Gamma)$ versus $(K)$ for three different values of the temperature of dust grain, and constant dust charge (Z)and plasma density $\left(N=10^{10} \mathrm{~m}^{-3}\right)$. It can be shown that the $(\Gamma)$ was decreased with increasing $\left(T_{d}\right)$ because of increasing in kinetic energy .Same reason can be said about for figures $(5,12)$. also it can be seen that the critical value of $(\Gamma)$ occurs at $\left(T_{\mathrm{d}}=\right.$ $0.02 \mathrm{eV}),(K \approx 0.4)$ and $\left(T_{d}=0.03 \mathrm{eV}\right),($ $K \approx 1.3$ ).

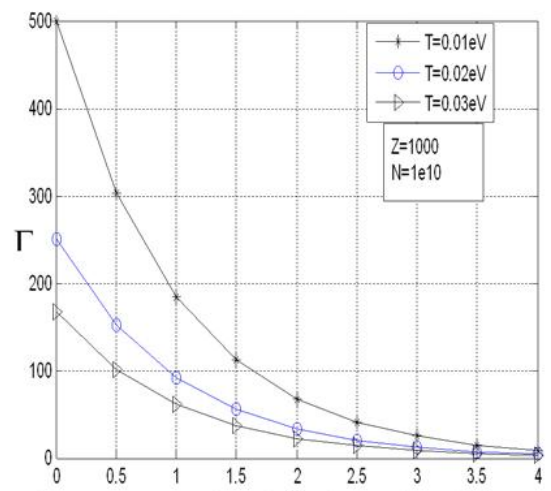

Fig.2 $(\Gamma)$ as a functiolt of $(K)$ for different value of $\left(T_{d}\right)$ 
Fig.(3) shows the $(\Gamma)$ as a function of $(K)$ for three different values of the plasma density, and constant $(Z$ )and ( $\left.T_{d}\right)$.It can be seen that the $(\Gamma)$ was increased with increasing of $(\mathrm{N})$. This is because increased $(\mathrm{N})$ mean decreasing the screening length $\mathrm{r}_{\mathrm{D}}=$ $\left(\varepsilon_{0} K T N e^{2}\right)^{1 / 2}[12]$ hence $(\Gamma)$ was increased. Critical value of $(\Gamma)$ occurs at $\left(N=10^{10} \mathrm{~m}^{-3}\right),(K \approx 0.2)$ and $(N=$ $\left.10^{11} m^{-3}\right),(K \approx 0.85)$.

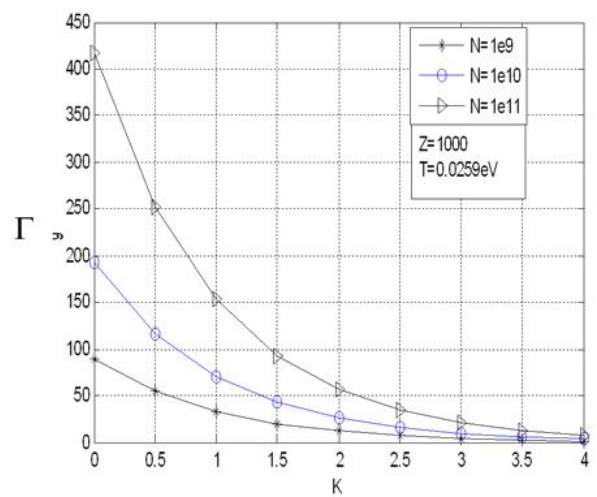

Fig. $3(\Gamma)$ as a function of $(K)$ for different value of $(N)$

Figures (4) shows the coulomb coupling parameter as a function of the grain charge, it can be seen that the $(\Gamma)$ was increased with increasing (Z) (square law).

Fig.(4) shows $(\Gamma)$ versus $(Z)$ for three different values of structure parameter $(K)$, and constant $\left(T_{d}\right.$ $=0.025 \mathrm{eV})$ and $\left(N=10^{10} \mathrm{~m}^{-3}\right)$, it can be shown that for given value of $(Z)$, that $(\Gamma)$ was increased with increasing $(K)$. This result was expect because of when the distance between the particles increase. The particles can move more freely i.e. increasing in its kinetic energy. Same reason can be said in $(7,10)$. Critical value of $(\Gamma)$ at $K=0.6, Z \approx 1300$ and $K=1, Z \approx 1550$.

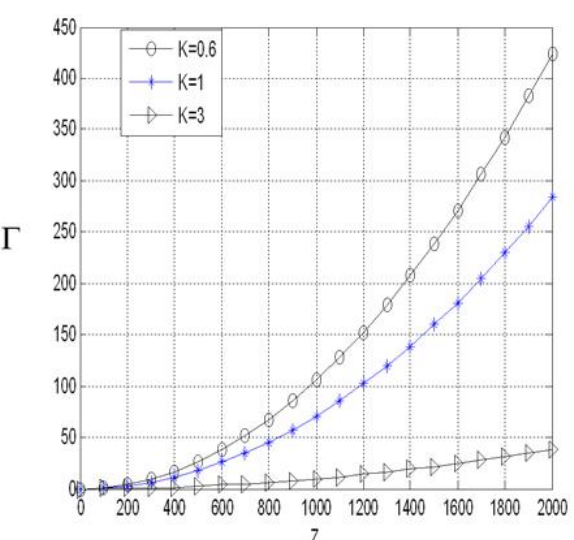

Fig. $4(\Gamma)$ as a function of $(Z)$ for different value of $(K)$.

Fig. (5) shows the $(\Gamma)$ as a function of $(Z)$, for three different values of structure parameter $(K)$, and constant $(K=2)$ and $\left(N=10^{10} \mathrm{~m}^{-3}\right)$, It can be shown that for given value of $(Z)$ the ( $\Gamma)$ was decreased with increasing $\left(T_{d}\right)$. The critical value of $(\Gamma)$ occurs at $\left(T_{d}=0.01 \mathrm{eV}\right),(\mathrm{Z} \approx 1600)$

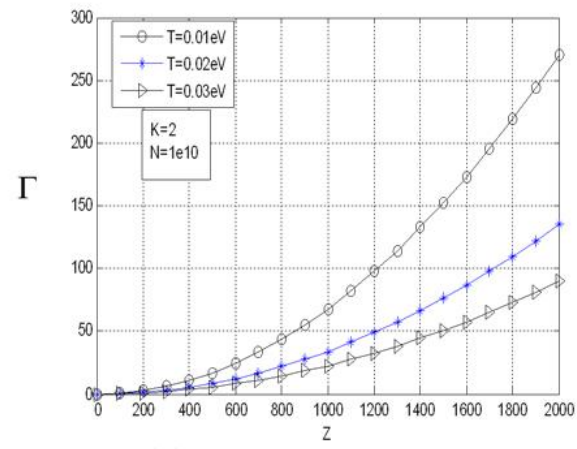

Fig. 5 the $(\Gamma)$ as a function of $(Z)$ for different value of $\left(T_{d}\right)$.

Fig.(6) shows the $(\Gamma)$ as a function of $(Z)$, for three different values of plasma density and constant $(K=2)$ and $\left(T_{d}=\right.$ $0.025 \mathrm{eV})$. It can be shown that for given value of $(Z)$ the $(\Gamma)$ was increased with increasing $(N)$. Critical value of $(\Gamma)$ occurs at $\left(N=10^{11}\right),(Z$ $\approx 1700$ ). 


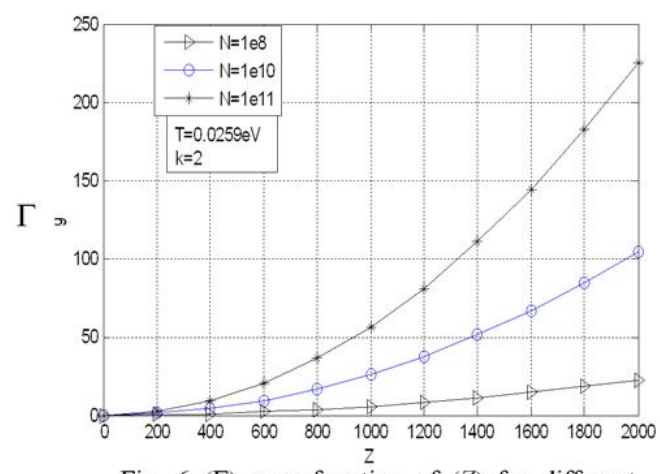

Fig. $6(\Gamma)$ as a function of $(Z)$ for different value of $(N)$.

Figures (7) shows the coulomb coupling parameter as a function of plasma density $(\mathrm{N})$.It can be shown that the $(\Gamma)$ was increased with $(N)$, also can be seen that the $(\Gamma)$ can be constant at a large value of $(N)$ less than $5 \times 10^{10} \mathrm{I} \mathrm{n}$ fig. 7,8 and beyond 5 $\times 10^{11}$ in fig.(9).

Fig. (7) for three different values of structure parameter $(K)$ and constant $(Z=1000)$ and $\left(T_{d}=0.025 \mathrm{eV}\right)$. It can be shown that for given value of $(N)$, that the $(\Gamma)$ was increased with decreasing $(K)$. Critical $(\Gamma)$ occurs at $K=0.2, N \approx$ $1.2 \times 10^{10}$.

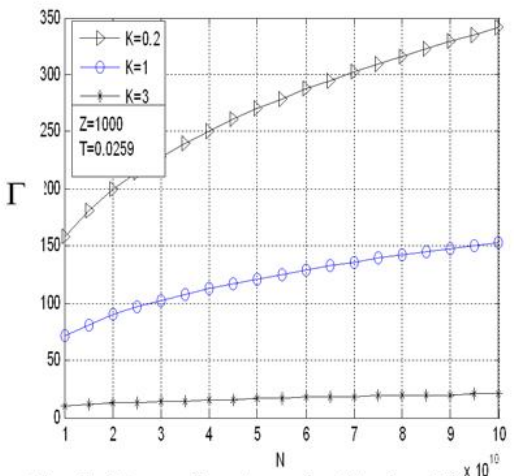

Fig. $7(\Gamma)$ as a function of a $(N)$ for different value of $(K)$.

Fig.(8) shows $(\Gamma)$ versus $(N)$ for three different values of grain charge $(Z)$ and constant $\left(T_{d}=0.025 \mathrm{eV}\right)$ and
$(K=2)$. It can be shown that for given value of $(N)$, that the $(\Gamma)$ was increased with increasing $(Z)$. Critical ( $\Gamma)$ occurs at $\left(N=2.3 \times 10^{11}\right),(Z=1500)$.

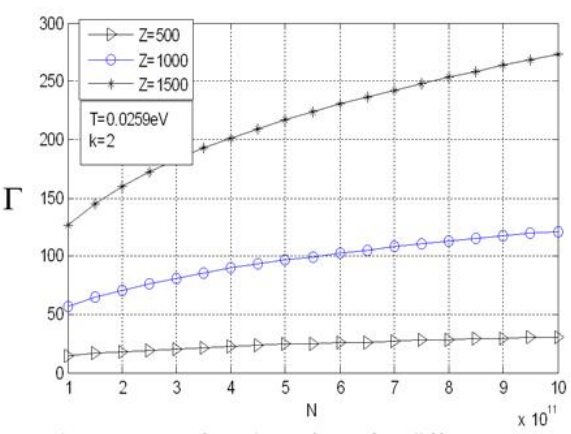

Fig. $8(\Gamma)$ as a function of $(N)$ for different value of $(Z)$

Fig.(9) shows ( $\Gamma$ ) versus $(\mathrm{N})$ for three different values of grain temperature $\left(T_{d}\right)$ and constant $(Z=1000)$ and $(K=2)$. It can be shown that for given value of $(\mathrm{N})$, that the $(\Gamma)$ was decreased with increasing $\left(\mathrm{T}_{\mathrm{d}}\right)$.Critical $(\Gamma)$ occurs at $\quad(N$ $\left.\approx 1.8 \times 10^{11} \mathrm{~m}^{-3}\right),(\mathrm{T}=0.01 \mathrm{eV})$

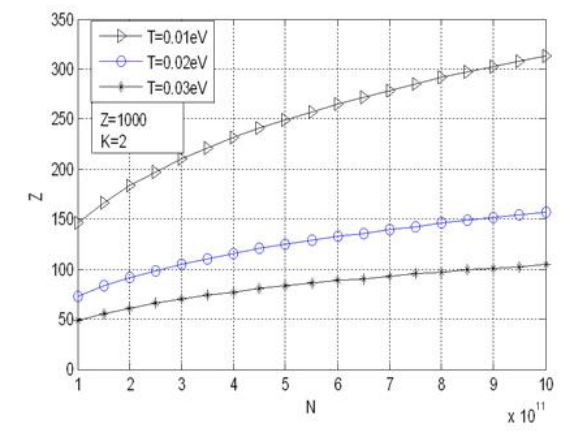

Fig. $9(\Gamma)$ as a function of $(N)$ for different value of $\left(T_{d}\right)$

Figures (10) shows the Coulomb coupling parameter as a function of the grain temperature $\left(T_{d}\right)$. It can be shown that the $(\Gamma)$ was decreased with Figures (10) shows the Coulomb coupling parameter as a function of the grain temperature $\left(T_{d}\right)$. It can be shown that the $(\Gamma)$ was decreased with 
increasing $\left(T_{d}\right)$, and when $\mathrm{T}_{\mathrm{d}}=0$ that the $(\Gamma)=\infty$ and also it can be shown that (Г) approach $\sim$ zero at a large value of (T).

Fig.(10) shows $(\Gamma)$ versus $\left(T_{d}\right)$ for three different values structure parameter $(K)$ and constant $(Z=1000)$ and $\left(N=10^{10} \mathrm{~m}^{-3}\right)$. shown that the $(\Gamma)$ was decreased with increasing $(K)$. Critical value of $(\Gamma)$ at $\left(T_{d} \approx 0.012 \mathrm{eV}\right.$, $K=1)$ and $\left(T_{d} \approx 0.0255 \mathrm{eV}, K=0.2\right)$.

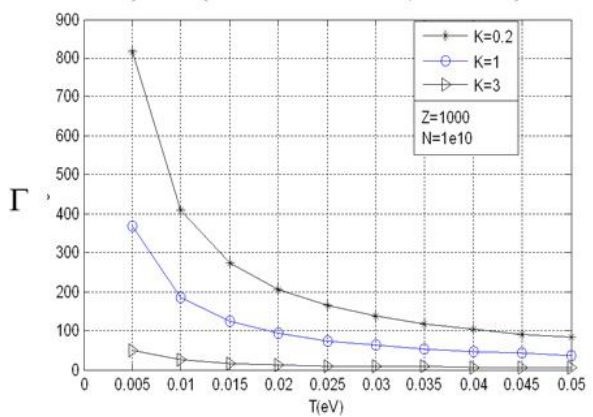

Fig. $10(\Gamma)$ as a function of $\left(T_{d}\right)$ for different value of $(K)$.

Fig.(11) shows $\Gamma$ versus $T_{d}$ for three different values grain charge $(Z)$ and constant $(K=2)$ and $\left(N=10^{10} \mathrm{~m}^{-3}\right)$. It can be shown that for given value of $\left(T_{d}\right)$ the $(\Gamma)$ was increased with increasing $(Z)$. Critical Value of $(\Gamma)$ at $\left(T_{d} \approx\right.$ $0.009 \mathrm{eV}),(Z=1500)$

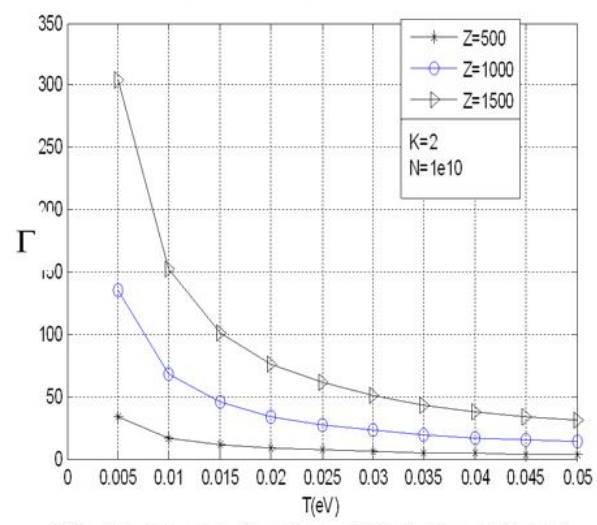

Fig. $11(\Gamma)$ as a function of $\left(T_{d}\right)$ for different value of $(Z)$.
Fig. 12 show $\Gamma$ versus $T_{d}$ for three different values plasma density $(N)$ and constant $(K=2)$ and $(Z=1000)$. it can be seen that for given value of $\left(T_{d}\right)$ that the $(\Gamma)$ was increased with increasing $(N)$. Critical value of $(\Gamma)$ accurse at

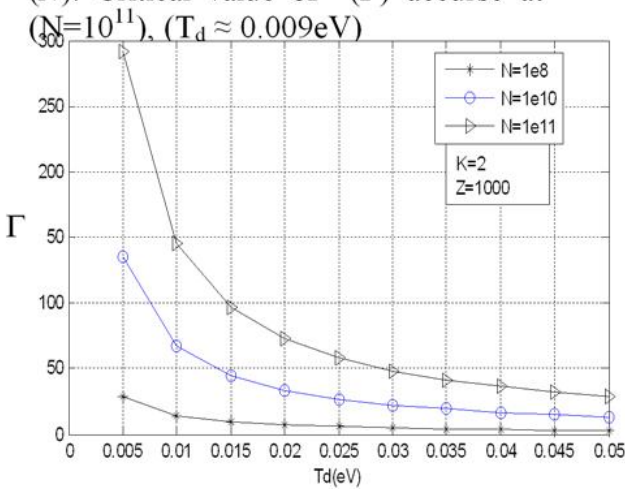

Fig. $12(\Gamma)$ as a function of $\left(T_{d}\right)$ for different value of $(\mathrm{N})$

\section{Reference}

1. Goertz .C. K, Rev.1989. Geophys. 27, 2711989

2. Merlino R. L. and Goree J. A. 2004. Physics Today, July

3. Shukla P. K. and Mamun A. A. 2002 Introduction to Dusty Plasma Physics, IOP, Bristol,

4. Merlino R. L. 2009 Chapter 5: Dusty Plasmas and Applications in Space and Industry,

in Plasma Physics Applied, Ed. C. Grabbe, Transworld Research, Kerala, India,

5. Shukla P K and Mamun A.A. 2002 Introduction to dusty plasma physics

6. Ichimaru S. Rev. Mod. Phys., 1982, 54, No. 4, 1017-1059.

7. Slattery W.L, Doolen G.D and Dewitt H.E. .1980. Improved equation of state foe the classical one-component plasma Phys. Rev. A, 21, 2087 
8. Hamaguchi S., Farouki R. T. J. Chem. Phys., 1994, 101, No 11, 9876-9884.

9. Farouche R. T., Hamaguchi S. J. Chem. Phys.. 1994. 101, No. 11, 9885-9893.

10. Hamaguchi S., Farouki R.T., Dubin D. H. E. J. Chem. Phys.1996. 105,No. 17, 7641-7647.
11. Vishnyakov V. I., Dragan G. S. Ukr. J. Phys.. 2004. 49, No. 2, 132136.

12. Francis F Chen .1988 . Plasma Physics and Controlled Fusion, Volum1:plasma physics

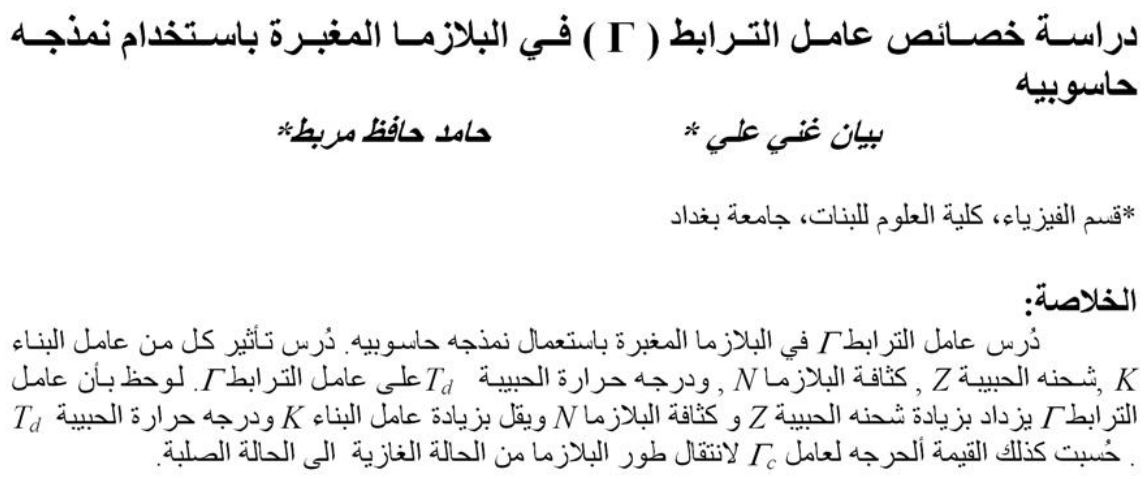

